\title{
K-isothermic hypersurfaces
}

\author{
Superfícies k-isotérmicas
}

\author{
Armando M. V. Corro * \\ Luciana Ávila Rodrigues ${ }^{\dagger}$ \\ Marcelo Lopes Ferro ${ }^{\ddagger}$
}

\begin{abstract}
We consider $n$ dimensional hypersurfaces in the Euclidean space and introduce the k-isothermic hypersurfaces, with $k<n$, as hypersurfaces that locally admit orthogonal parametrization by curvature lines with $\mathrm{k}$ distinct coefficients of the first fundamental form. It easy to check that the transformations: isometries, dilations and invertions, preserve k-isothermic hypersurfaces. We prove that there are no k-isothermic hypersurface of dimension $n$ with distinct principal curvatures for $n \geq k+3$. We introduced two ways to generate a $(\mathrm{k}+1)$-isothermic hypersurface from a k-isothermic hypersurfaces, which we will call 2-reducible. Moreover, we provide a local characterization of Dupin 2-isothermic hypersurfaces and include explicit examples of 2-irreducible Dupin 2-isothermic hypersurfaces.
\end{abstract}

Keywords: k-isothermic hypersurfaces. Dupin hypersurfaces. Irreducible hypersurface.

Resumo: Consideramos hipersuperfícies de dimensão $n$ no espaço Euclidiano e introduzimos as hipersuperfícies k-isotérmicas, com $k<n$, como hipersuperfícies que localmente admitem parametrizações ortogonais por linhas de curvatura com k coeficientes da primeira forma quadrática distintos. É fácil ver que as transformações: isometrias, dilatações e inversões, preservam hipersuperfícies k-isotérmicas. Provamos que não existem hipersuperfícies k-isotérmica de dimensão $n$ com curvaturas principais distintas para $n \geq k+3$. Introduzimos duas maneiras para gerar uma hipersuperfície $(k+1)$-isotérmica apartir de uma hipersuperfície k-isotérmica, a qual chamamos 2-redutível. Além disso, apresentamos uma

*Instituto de Matemática e Estatística, Universidade Federal de Goiás, corro@ufg.br †Departamento de Matemática, Universidade de Brasília, luavila@unb.br

¥Instituto de Matemática e Estatística, Universidade Federal de Goiás, marceloferro@ufg.br 
Corro, A. M. V.; Ferro, M. L.; Rodrigues, L. A.

caracterização local para as hipersuperfícies de Dupin 2-isotérmicas e incluímos exemplos explícitos de tais hipersuperfícies 2-irredutíveis.

Palavras-chave: Hipersuperfície k-isotérmica. Hipersuperfície de Dupin. Hipersuperfícies irredutível.

\section{Introduction}

The investigation of conformally flat hypersurfaces has been of interest for quite some time. Any surface in $R^{3}$ is conformally flat, since it can be parametrized by isothermic coordinates. For higher dimensional hypersurfaces, E. Cartan [3] gave a complete classification for the conformally flat hypersurfaces of $n+1$ dimensional space form when $n \geq 4$. He proved that such hypersurfaces are quasi-umbilic, i.e., one of the principal curvatures has multiplicity at least $n-1$. In the same paper, Cartan investigated the case $n=3$. He showed that the quasi-umbilical hypersurfaces are conformally flat, but the conversely does not hold (for a proof see [17]). Moreover, he gave a characterization of the conformally flat 3-dimensional hypersurfaces, with three distinct principal curvatures, in therms of certain integrable distributions. A classification of 3 dimensional hypersurfaces is a current research topic, some works in this direction are [3], [14] - [17], [24] - [27].

A particular class of conformally flat hypersurfaces are the called isothermic hypersurfaces, which locally allow parameterization by curvature lines with all coefficients of the first quadratic form equal. All isothermic hypersurface is a conformally flat hypersurface, but the conversely does not hold. In the case of surfaces in $R^{3}$ the classification is an open problem, some works in this direction are [2], [5] - [11]. Particular classes of isothermic surfaces are, the constant mean curvature surfaces, quadrics surfaces [28], surfaces whose lines of curvature has constant geodesic curvature, in particular, the cyclides of Dupin. Case $n=3$ is studied as a particular case in studies dealing with conformally flat hypersurfaces.

A hypersurface $M$, immersed into a space form, is said to be Dupin if each principal curvature is constant along its corresponding line or surface of curvature. $M$ is a proper Dupin hypersurface if the number $g$ of distinct principal curvatures is constant on $M$. Dupin surfaces were first studied by Dupin in 1822. Pinkall [18], studied Dupin hypersurfaces in the setting of Lie sphere geometry. The local theory of Dupin hypersurfaces in $R^{n}$ or $S^{n}$ or $H^{n}$ is essentially the same.

In this paper, we define the $n$ dimensional k-isothermic hypersurfaces in the eu-

NEXUS Mathematicæ, Goiânia, v. 3, 2020, e20004. 
clidean space, with $k<n$, as hypersurfaces that locally admit orthogonal parametrization by curvature lines with $\mathrm{k}$ distinct coefficients of the first quadratic form. Such hypersurfaces are preserved by isometries, dilations and invertions. We prove that there are no $n$ dimensional k-isothermic hypersurface with distinct principal curvatures for $n \geq k+3$. We introduced two ways to generate a $(\mathrm{k}+1)$-isothermic hypersurface from a k-isothermic hypersurface, which we will call 2-reducible. Moreover, we provide a local characterization of Dupin 2-isothermic hypersurface in $R^{4}$ and $R^{5}$ and include explicit examples of 2-isothermic hypersurface irreducible.

The paper is organized as follows. In sect. 2, we define k-isothermic hypersurfaces in a local coordinate system and we give some properties of hypersurfaces parametrized by lines of curvature, with distinct principal curvatures, that will be used in the next section. In sect. 3, we show that there is no k-isothermic hypersurface in $R^{n+1}$ parametrized by lines of curvature, if $n \geq k+3$. In sect. 4, we give a local characterization of Dupin hypersurfaces in $R^{4}$ and $R^{5}$ that are 2-isothermic. We finish by presenting examples of such a 2 -isothermic Dupin hypersurfaces in $R^{5}$ which are irreducible and have nonconstant Lie curvature.

\section{Properties of hypersurfaces with distinct princi- pal curvatures}

An immersion $X: \Omega \subseteq R^{n} \rightarrow R^{n+1}$ is a k-isothermic hypersurface if the first fundamental form is given by

$$
d s^{2}=\sum_{i=1}^{k} L_{i}\left(d x_{\alpha_{i}+1}^{2}+\ldots+d x_{\alpha_{i}+m_{i}}^{2}\right),
$$

with $\alpha_{1}=0$ and for $2 \leq l \leq k, \alpha_{l}=\sum_{j=1}^{l-1} m_{j}$, where $m_{i}$ are called multiplicity of coefficients $L_{i}, 1 \leq i \leq k$, with $m_{1}+m_{2}+\ldots+m_{k}=n$.

An hypersurface $M$ is a $k$-isothermic hypersurface if locally can be parametrized by an immersion k-isothermic.

Let $X: \Omega \subseteq R^{n} \rightarrow R^{n+1}, X\left(x_{1}, \ldots, x_{n}\right)$ be a hypersurface parametrized by lines of curvature, with distinct principal curvatures, $-\lambda_{i}, 1 \leq i \leq n$, and let $N: \Omega \subseteq R^{n} \rightarrow R^{n+1}$ be a unit normal vector field of X. Then 


$$
<X,,_{i}, X,_{j}>=\delta_{i j} g_{i j}, \quad N,_{i}=\lambda_{i} X,_{i} \quad 1 \leq i \neq j \leq n,
$$

where $<,>$ denotes the Euclidean metric on $R^{n+1}$.

Moreover for $1 \leq i \neq j \leq n$, we have

$$
\begin{aligned}
& X,{ }_{i j}-\Gamma_{i j}^{i} X,_{i}-\Gamma_{i j}^{j} X,{ }_{j}=0, \\
& \Gamma_{i j}^{i}=\frac{\lambda_{i, j}}{\lambda_{j}-\lambda_{i}},
\end{aligned}
$$

where $\Gamma_{i j}^{i}$ are the Christoffel symbols, given in terms of the metric (2) by

$$
\Gamma_{i j}^{k}=0, \quad \Gamma_{i i}^{i}=\frac{g_{i i, i}}{2 g_{i i}}, \quad \Gamma_{i i}^{j}=-\frac{g_{i i, j}}{2 g_{j j}}, \quad \Gamma_{i j}^{i}=\frac{g_{i i, j}}{2 g_{i i}},
$$

where $i, j, k$ are distinct. It follows from (5) that

$$
\begin{aligned}
g_{i i, j}=2 \Gamma_{i j}^{i} g_{i i}, & 1 \leq i \neq j \leq n, \\
\Gamma_{i i}^{j}=-\Gamma_{i j}^{i} \frac{g_{i i}}{g_{j j}}, & 1 \leq i \neq j \leq n .
\end{aligned}
$$

From (2) and (6), we get

$$
X,_{i i}=\Gamma_{i i}^{i} X,_{i}-\sum_{k \neq i} \Gamma_{i k}^{i} \frac{g_{i i}}{g_{k k}} X,_{k}-\lambda_{i} g_{i i} N .
$$

For a hypersurfaces with distinct principal curvatures, the Lie curvature is defined by

$$
\Psi^{j k l i}=\frac{\left(\lambda_{j}-\lambda_{k}\right)\left(\lambda_{l}-\lambda_{i}\right)}{\left(\lambda_{j}-\lambda_{i}\right)\left(\lambda_{l}-\lambda_{k}\right)}, \quad i, j, k, l \text { distinct } .
$$

Proposition 1. [13]. Let $X: \Omega \subseteq R^{n} \rightarrow R^{n+1}$, be a hypersurface parametrized by lines of curvature, whose principal curvatures $-\lambda_{i}, 1 \leq i \leq n$, are distinct. Then the Gauss equation for the immersion $X$ is given by

$$
\lambda_{i} \lambda_{j}+\frac{L_{i j}}{g_{i i}}+\frac{L_{j i}}{g_{j j}}+\sum_{k \neq i, j} \frac{\Gamma_{i k}^{i} \Gamma_{j k}^{j}}{g_{k k}}=0, \quad i \neq j
$$


where

$$
L_{i j}=\Gamma_{i j, i}^{j}-\Gamma_{i j}^{j}\left(\Gamma_{i i}^{i}-\Gamma_{i j}^{j}\right)
$$

Using (5), we obtain immediately

Proposition 2. Let $X: \Omega \subseteq R^{n} \rightarrow R^{n+1}$, be a $k$-isothermic hypersurface parametrized by lines of curvature, with $n$ distinct principal curvatures. Then the Christoffel symbols (5) satisfy for $\alpha_{l}+1 \leq i, j \leq \alpha_{l}+m_{l}, \alpha_{r}+1 \leq a, b \leq \alpha_{r}+m_{r}$ and $1 \leq s \leq n$,

$$
\Gamma_{i s}^{i}=\Gamma_{j s}^{j}, \quad \Gamma_{a s}^{a}=\Gamma_{b s}^{b}, \quad \Gamma_{i i}^{i}=\Gamma_{j i}^{j}, \quad \Gamma_{a a}^{a}=\Gamma_{b a}^{b}
$$

where $\alpha_{l}, \alpha_{r}, m_{l}$ and $m_{r}$ with $1 \leq l \neq r \leq k$, are given by (1).

An immersion $X: \Omega \subseteq R^{n} \rightarrow R^{n+1}$ is a Dupin hypersurface if each principal curvature is constant along its corresponding line ( or surface ) of curvature. If the multiplicity of the principal curvatures is constant then the Dupin submanifold is said to be proper.

\section{$3 \quad$ K-isothermic hypersurfaces}

In this section, we show that there is no k-isothermic hypersurface parametrized by lines of curvature, if $n \geq k+3$. This is achieved by the following theorem.

Theorem 3. There is no $M^{n} \subseteq R^{n+1} k$-isothermic hypersurface, with $n$ distinct principal curvatures for $n \geq k+3$.

Proof: Suppose that $M^{n} \subseteq R^{n+1}$ be a k-isothermic hypersurface. Let $X: \Omega \subseteq$ $R^{n} \rightarrow R^{n+1}$ be a parametrization by lines of curvature for $M$ and denote by $-\lambda_{i}$ the distinct principal curvatures. As $k<n$, it follows from (1) that there is $1<m_{i_{0}}<n$, such that the first fundamental form of $X$, is given by

$$
d s^{2}=L_{i_{0}}\left(d x_{\alpha_{i_{0}}+1}^{2}+\ldots+d x_{\alpha_{i_{0}}+m_{i_{0}}}^{2}\right)+\sum_{i \neq i_{0}}^{k} L_{i}\left(d x_{\alpha_{i}+1}^{2}+\ldots+d x_{\alpha_{i}+m_{i}}^{2}\right)
$$

with $\alpha_{1}=0$ and for $2 \leq l \leq k, \alpha_{l}=\sum_{j=1}^{l-1} m_{j}$, where $m_{1}+m_{2}+\ldots+m_{k}=n$. 
We will prove that $1<m_{i_{0}} \leq 3$. In fact, suppose $m_{i_{0}} \geq 4$, then using (9) and (10), for $\alpha_{i_{0}}+1 \leq i, j, k, l \leq \alpha_{i_{0}}+m_{i_{0}}$ distinct, we have

$$
\begin{aligned}
& \lambda_{i} \lambda_{j}=\frac{-1}{L_{i_{0}}}\left[\Gamma_{i j, i}^{j}+\Gamma_{i j, j}^{i}+\left(\Gamma_{i k}^{i}\right)^{2}+\left(\Gamma_{i l}^{i}\right)^{2}\right]-\sum_{s \neq i, j, k, l}^{n} \frac{\left(\Gamma_{i s}^{i}\right)^{2}}{L_{s}}, \\
& \lambda_{i} \lambda_{k}=\frac{-1}{L_{i_{0}}}\left[\Gamma_{i j, i}^{j}+\Gamma_{i k, k}^{i}+\left(\Gamma_{i j}^{i}\right)^{2}+\left(\Gamma_{i l}^{i}\right)^{2}\right]-\sum_{s \neq i, j, k, l}^{n} \frac{\left(\Gamma_{i s}^{i}\right)^{2}}{L_{s}}, \\
& \lambda_{l} \lambda_{j}=\frac{-1}{L_{i_{0}}}\left[\Gamma_{l j, l}^{j}+\Gamma_{i j, j}^{i}+\left(\Gamma_{i k}^{i}\right)^{2}+\left(\Gamma_{i l}^{l}\right)^{2}\right]-\sum_{s \neq i, j, k, l}^{n} \frac{\left(\Gamma_{l s}^{l}\right)^{2}}{L_{s}}, \\
& \lambda_{l} \lambda_{k}=\frac{-1}{L_{i_{0}}}\left[\Gamma_{l j, l}^{j}+\Gamma_{i k, k}^{i}+\left(\Gamma_{i j}^{i}\right)^{2}+\left(\Gamma_{i l}^{l}\right)^{2}\right]-\sum_{s \neq i, j, k, l}^{n} \frac{\left(\Gamma_{l s}^{l}\right)^{2}}{L_{s}} .
\end{aligned}
$$

Subtracting, respectively, in (11) the first and second equation, and the third and last equation, we obtain

$$
\begin{aligned}
& \lambda_{i}\left(\lambda_{j}-\lambda_{k}\right)=\frac{-1}{L_{i_{0}}}\left[\Gamma_{i j, j}^{i}-\Gamma_{i k, k}^{i}+\left(\Gamma_{i k}^{i}\right)^{2}-\left(\Gamma_{i j}^{i}\right)^{2}\right], \\
& \lambda_{l}\left(\lambda_{j}-\lambda_{k}\right)=\frac{-1}{L_{i_{0}}}\left[\Gamma_{i j, j}^{i}-\Gamma_{i k, k}^{i}+\left(\Gamma_{i k}^{i}\right)^{2}-\left(\Gamma_{i j}^{i}\right)^{2}\right] .
\end{aligned}
$$

Therefore we obtained one contradiction, because all principal curvatures are distinct. Therefore $1<m_{i_{0}} \leq 3$.

Now we will prove that for $j_{0} \neq i_{0}, m_{j_{0}}=1$. Suppose $m_{j_{0}}>1$, then rewritten the first fundamental form of $X$ as

$$
\begin{aligned}
d s^{2}=\quad & L_{i_{0}}\left(d x_{\alpha_{i_{0}}+1}^{2}+\ldots+d x_{\alpha_{i_{0}}+m_{i_{0}}}^{2}\right)+L_{j_{0}}\left(d x_{\alpha_{j_{0}}+1}^{2}+\ldots+d x_{\alpha_{j_{0}}+m_{j_{0}}}^{2}\right)+ \\
& \sum_{i \neq i_{0}, j_{0}}^{k} L_{i}\left(d x_{\alpha_{i}+1}^{2}+\ldots+d x_{\alpha_{i}+m_{i}}^{2}\right)
\end{aligned}
$$

and using (9) and (10), for $\alpha_{i_{0}}+1 \leq i \neq j \leq \alpha_{i_{0}}+m_{i_{0}}$ and $\alpha_{j_{0}}+1 \leq k \neq l \leq \alpha_{j_{0}}+m_{j_{0}}$, 
we have

$$
\begin{aligned}
& \lambda_{i} \lambda_{k}=\frac{-L_{i k}}{L_{i_{0}}}-\frac{L_{k i}}{L_{j_{0}}}-\frac{\Gamma_{i j}^{i} \Gamma_{k j}^{k}}{L_{i_{0}}}-\frac{\Gamma_{i l}^{i} \Gamma_{k l}^{k}}{L_{j_{0}}}-\sum_{s \neq i, j, k, l}^{n} \frac{\Gamma_{i s}^{i} \Gamma_{k s}^{k}}{L_{s}}, \\
& \lambda_{i} \lambda_{l}=\frac{-L_{i l}}{L_{i_{0}}}-\frac{L_{l i}}{L_{j_{0}}}-\frac{\Gamma_{i j}^{i} \Gamma_{k j}^{k}}{L_{i_{0}}}-\frac{\Gamma_{i k}^{i} \Gamma_{k l}^{l}}{L_{j_{0}}}-\sum_{s \neq i, j, k, l}^{n} \frac{\Gamma_{i s}^{i} \Gamma_{k s}^{k}}{L_{s}} \\
& \lambda_{j} \lambda_{k}=\frac{-L_{j k}}{L_{i_{0}}}-\frac{L_{k j}}{L_{j_{0}}}-\frac{\Gamma_{i j}^{j} \Gamma_{k i}^{k}}{L_{i_{0}}}-\frac{\Gamma_{i l}^{i} \Gamma_{k l}^{k}}{L_{j_{0}}}-\sum_{s \neq i, j, k, l}^{n} \frac{\Gamma_{i s}^{i} \Gamma_{k s}^{k}}{L_{s}}, \\
& \lambda_{j} \lambda_{l}=\frac{-L_{j l}}{L_{i_{0}}}-\frac{L_{l j}}{L_{j_{0}}}-\frac{\Gamma_{i j}^{j} \Gamma_{k i}^{k}}{L_{i_{0}}}-\frac{\Gamma_{i k}^{i} \Gamma_{k l}^{l}}{L_{j_{0}}}-\sum_{s \neq i, j, k, l}^{n} \frac{\Gamma_{i s}^{i} \Gamma_{k s}^{k}}{L_{s}} .
\end{aligned}
$$

Again, subtracting the two last equations and two first equations, using (10) and expression of $L_{r s}$ given by (9), we obtain $\left(\lambda_{i}-\lambda_{j}\right)\left(\lambda_{l}-\lambda_{k}\right)=0$, which is a contradiction since all principal curvatures are pairwise distinct. Therefore $m_{j_{0}}=1$.

Therefore, for the existence of $M$, we must have $m_{i_{0}}=2$ or $m_{i_{0}}=3$ and for all $j \neq i_{0}, m_{j}=1$. Thus

If $m_{i_{0}}=2$, then for all $j \neq i_{0}, m_{j}=1$ and so $n=2+k-1=1+k$.

If $m_{i_{0}}=3$, then for all $j \neq i_{0}, m_{j}=1$ and so $n=3+k-1=2+k$.

Thus, for $M \mathrm{k}$-isothermic to exist, we must have $n=k+1$ or $n=k+2$. Therefore, if $n \geq k+3$, then there is no $M$ k-isothermic.

Proceeding analogously to obtaining reducible Dupin hypersurface (for more details, see [18]), we define 2-reducible k-isothermic hypersurface.

Let $M^{n} \subseteq R^{n+1}$ be a k-isothermic hypersurface, with coefficients $L_{i}, 1 \leq i \leq k$ not constant. It is then easy to check that the following constructions lead to a $(\mathrm{k}+1)$-isothermic hypersurface $\widetilde{M}$ in $R^{n+2}$ :

(1) Let $\widetilde{M}$ be the cylinder $M \times R \subseteq R^{n+2}$.

(2) Project $M$ stereographically onto a hypersurface $\widehat{M} \subseteq S^{n+1}$. Let $\widetilde{M}$ be the cone R. $\widehat{M}$ over $\widehat{M}$.

We define a 2-reducible $k$-isothermic hypersurface as a k-isothermic hypersurface obtained by one of the two above constructions. More generally, all locally k-isothermic hypersurfaces obtained by isometries, dilations and inversions in a hypersurface will also be called 2-reducible. A hypersurface is a 2-irreducible k-isothermic hypersur- 
face if it is not 2-reducible.

Remark 4. We observed that in [18] the author presents two more constructions that preserve Dupin hypersurfaces.

(3) Take an n dimensional linear subspace $R^{n} \subseteq R^{n+1}$ and consider the rotations $\phi_{t}$

of $R^{n+2}$ that leave $R^{n}$ pointwise fixed. Let $\widetilde{M}$ be the hypersurface of $R^{n+2}$ generated by $M$ under the rotations $\phi_{t}$.

(4) Let $\widetilde{M}$ be a tube in $R^{n+2}$ around $M \subseteq R^{n+1}$.

A hypersurface is said to be reducible if it is obtained by one of the four above constructions, and every Dupin hypersurface that is locally Lie equivalent to such a hypersurface. The third and fourth construction does not generate a $(k+1)$ isothermic hypersurface.

\section{Class of 2-isothermic Dupin hypersurfaces parame- trized by lines of curvature}

In the previous section, we show that there is no k-isothermic hypersurface parametrized by lines of curvature, if $n \geq k+3$. Using the 2 -reducible definition we can construction examples of 2-isothermic Dupin hypersurfaces.

Example 5. Let $M^{2} \subseteq R^{3}$ be a Dupin isothermic surface, with two non zero distinct principal curvatures. Then the cylinder $M^{2} \times R \subseteq R^{4}$ is a 2 -isothermic Dupin hypersurface with three distinct principal curvatures, two of them given by the principal curvatures of $M^{2}$ and the third one is given by the null function.

We characterize the Dupin hypersurfaces that are 2-isothermic in $R^{4}$ and $R^{5}$. Using the Theorem 3 , where for simplicity of notation we make $i_{0}=1$ we have two cases, which without loss of generality are:

Case 1: $m_{1}=2$ i.e, the Dupin hypersurface that is 2 -isothermic in $R^{4}$ has the first fundamental form

$$
d s^{2}=L_{1}\left(d x_{1}^{2}+d x_{2}^{2}\right)+L_{2} d x_{3}^{2} .
$$

Case 2: $m_{1}=3$ i.e, the Dupin hypersurface that is 2-isothermic in $R^{5}$ has the first 
fundamental form

$$
d s^{2}=L_{1}\left(d x_{1}^{2}+d x_{2}^{2}+d x_{3}^{2}\right)+L_{2} d x_{4}^{2} .
$$

We will show that there exist such hypersurfaces by presenting examples.

The following lemma provides a characterization of the principal curvatures of a Dupin hypersurface from Case 1.

Lemma 6. Let $M^{3} \subseteq R^{4}$, be a proper Dupin hypersurface that is 2-isothermic of the Case 1 type and that has three distinct principal curvatures. Then the principal curvatures are given by

$$
\begin{aligned}
& \lambda_{1}=f_{2} h_{3}+h_{2}, \\
& \lambda_{2}=f_{1} h_{3}+h_{1}, \\
& \lambda_{3}=\frac{f_{2} \lambda_{2}-f_{1} \lambda_{1}}{f_{2}-f_{1}} .
\end{aligned}
$$

Moreover, there is a change in each coordinate separately such that the coefficients $L_{1}$ and $L_{2}$ of the first fundamental form (13) are given by

$$
L_{1}=\frac{1}{\left(\lambda_{2}-\lambda_{1}\right)^{2}}, \quad L_{2}=\left[\frac{f_{2}-f_{1}}{\lambda_{2}-\lambda_{1}}\right]^{2} .
$$

Where $f_{i}$ and $h_{j}$ are, respectively, differentiable functions of $x_{i}$ and $x_{j}, 1 \leq i \leq 2$, $1 \leq j \leq 3$.

Proof: We will first show that each $\lambda_{i}$ is given by (15). Using (10), we have

$$
\Gamma_{13}^{1}=\Gamma_{23}^{2} .
$$

In this equation, using (4), we obtain

$$
\left(\frac{\lambda_{3}-\lambda_{2}}{\lambda_{3}-\lambda_{1}}\right)_{, 3}=0
$$


Moreover, differentiating $\frac{\lambda_{3}-\lambda_{2}}{\lambda_{3}-\lambda_{1}}$, with respect to $x_{1}$ and $x_{2}$, we get

$$
\left(\log \frac{\lambda_{3}-\lambda_{2}}{\lambda_{3}-\lambda_{1}}\right)_{, 12}=0
$$

Using (17) and (18), we obtain

$$
\frac{\lambda_{3}-\lambda_{2}}{\lambda_{3}-\lambda_{1}}=\frac{f_{1}}{f_{2}}
$$

where $f_{1}$ and $f_{2}$ are, respectively, differentiable functions of $x_{1}$ and $x_{2}$.

From (19), we have

$$
\left(\lambda_{3}-\lambda_{2}\right) f_{2}=\left(\lambda_{3}-\lambda_{1}\right) f_{1} .
$$

Differentiating this equation with respect to $x_{3}$, we get

$$
\lambda_{2,3} f_{2}=\lambda_{1,3} f_{1}
$$

Now, differentiating (21) with respect to $x_{2}$ and $x_{1}$, respectively, we obtain

$$
\begin{aligned}
& \lambda_{1,23}-\frac{f_{2}^{\prime}}{f_{2}} \lambda_{1,3}=0, \\
& \lambda_{2,13}-\frac{f_{1}^{\prime}}{f_{1}} \lambda_{2,3}=0 .
\end{aligned}
$$

Therefore integrating this two equations and using (21) and (19), we obtain (15). We show (16) by comparing (4) and (5), we get

$$
\frac{\lambda_{1,2}}{\lambda_{2}-\lambda_{1}}=\frac{L_{1,2}}{2 L_{1}}, \quad \frac{\lambda_{2,1}}{\lambda_{1}-\lambda_{2}}=\frac{L_{1,1}}{2 L_{1}}, \quad \frac{\lambda_{3,1}}{\lambda_{1}-\lambda_{3}}=\frac{L_{2,1}}{2 L_{2}},
$$

so that

$$
\left[L_{1}\left(\lambda_{2}-\lambda_{1}\right)^{2}\right]_{, 2}=0, \quad\left[L_{1}\left(\lambda_{2}-\lambda_{1}\right)^{2}\right]_{, 1}=0, \quad\left[L_{2}\left(\lambda_{3}-\lambda_{1}\right)^{2}\right]_{, 1}=0,
$$

and therefore

$$
L_{1}=\left[\frac{\bar{f}_{3}\left(x_{3}\right)}{\lambda_{2}-\lambda_{1}}\right]^{2}, \quad L_{2}=\left[\frac{\bar{f}_{23}\left(x_{2}, x_{3}\right)}{\lambda_{3}-\lambda_{1}}\right]^{2},
$$

where $\bar{f}_{3}$ is an arbitrary function of the variable $x_{3}$ and $\bar{f}_{23}$ is an arbitrary function 
of the variables $x_{2}$ and $x_{3}$.

Differentiating the first equations (23) with respect to $x_{3}$, using (10), (4) and (5), we have $\bar{f}_{3}^{\prime}\left(x_{3}\right)=0$. Therefore the first equation (23), can be rewritten as

$$
L_{1}=\left[\frac{c}{\lambda_{2}-\lambda_{1}}\right]^{2}
$$

where $c$ is a constant.

Differentiating the second equation of (23) with respect to $x_{2}$, using (4), we have

$$
L_{2,2}=2 L_{2}\left[\frac{\bar{f}_{23,2}}{\bar{f}_{23}}-\Gamma_{32}^{3} \frac{\lambda_{2}-\lambda_{3}}{\lambda_{3}-\lambda_{1}}+\Gamma_{12}^{1} \frac{\lambda_{2}-\lambda_{1}}{\lambda_{3}-\lambda_{1}}\right] .
$$

In this last equation, using (5), (4) and simplifying, we have

$$
\begin{aligned}
\frac{\bar{f}_{23,2}}{\bar{f}_{23}} & =\frac{\lambda_{2}-\lambda_{1}}{\lambda_{3}-\lambda_{1}}\left(\Gamma_{32}^{3}-\Gamma_{12}^{1}\right), \\
& =\frac{\lambda_{2}-\lambda_{1}}{\lambda_{3}-\lambda_{1}}\left(\log \frac{\lambda_{2}-\lambda_{1}}{\lambda_{2}-\lambda_{3}}\right)_{, 2} .
\end{aligned}
$$

Now, using (19), we have $\frac{\bar{f}_{23,2}}{\bar{f}_{23}}=\frac{f_{2}^{\prime}}{f_{2}}$. Therefore $\bar{f}_{23}=f_{2} f_{3}$ and using again (19) the second equation (23), can be rewritten as

$$
L_{2}=\left[\left(f_{2}-f_{1}\right) \frac{f_{3}}{\lambda_{2}-\lambda_{1}}\right]^{2}
$$

Thus using (24), (25) and making $d \widetilde{x}_{1}=c d x_{1}, d \widetilde{x}_{2}=c d x_{2}$ and $d \widetilde{x}_{3}=f_{3} d x_{3}$, we obtain (16).

The following lemma provides a characterization of the principal curvatures of a Dupin hypersurface from case 2.

Lemma 7. Let $M^{4} \subseteq R^{5}$, be a proper Dupin hypersurface that is 2-isothermic of the Case 2 type and that has four distinct principal curvatures. Then the principal 
curvatures are given by

$$
\begin{aligned}
& \lambda_{1}=\left(f_{2}+f_{3}\right) h_{4}+h_{3}+h_{2}, \\
& \lambda_{2}=c\left[\left(f_{1}+f_{3}\right) h_{4}+h_{3}+h_{1}\right], \\
& \lambda_{3}=\frac{c}{1-c}\left[\left(f_{1}-f_{2}\right) h_{4}+h_{1}-h_{2}\right], \\
& \lambda_{4}=\frac{c\left[\left(f_{2}-f_{1}\right) h_{3}-\left(h_{2}-h_{1}\right) f_{3}+f_{2} h_{1}-f_{1} h_{2}\right]}{f_{2}-c f_{1}+(1-c) f_{3}} .
\end{aligned}
$$

Moreover, there is a change in each coordinate separately such that the coefficients $L_{1}$ and $L_{2}$ of the first fundamental form (14) are given by

$$
L_{1}=\frac{1}{\left(\lambda_{2}-\lambda_{1}\right)^{2}}, \quad L_{2}=\left[\frac{f_{2}-c f_{1}+(1-c) f_{3}}{\lambda_{2}-\lambda_{1}}\right]^{2} \text {. }
$$

Where $f_{i}$ and $h_{j}$ are, respectively, differentiable functions of $x_{i}$ and $x_{j}, 1 \leq i \leq 3$, $1 \leq j \leq 4$, and $c \neq 1$ is a constant.

Proof: We will first show that each $\lambda_{i}$ is given by (26). By using (10), we have

$$
\begin{aligned}
& \Gamma_{13}^{1}=\Gamma_{23}^{2}, \quad \Gamma_{13}^{3}=\Gamma_{21}^{2}, \quad \Gamma_{12}^{1}=\Gamma_{23}^{3}, \\
& \Gamma_{14}^{1}=\Gamma_{24}^{2}=\Gamma_{34}^{3} .
\end{aligned}
$$

Using (28) and (29), we obtain

$$
\frac{\lambda_{3}-\lambda_{2}}{\lambda_{3}-\lambda_{1}}=c
$$

where $c \neq 1$ is constant.

Using (29), and (30), we have

$$
\frac{\lambda_{4}-\lambda_{2}}{\lambda_{4}-\lambda_{1}}=\frac{c h_{13}}{h_{23}}, \quad \frac{\lambda_{4}-\lambda_{3}}{\lambda_{4}-\lambda_{1}}=\frac{c h_{12}}{(1-c) h_{23}},
$$

where $h_{i 3}=f_{i}+f_{3}, 1 \leq i \leq 2, h_{12}=f_{1}-f_{2}$ and $f_{j}$ are differentiable functions of $x_{j} 1 \leq j \leq 3$. 
Using (30) and (31) we get that $\lambda_{1}$ and $\lambda_{2}$ satisfying

$$
\begin{array}{cl}
\lambda_{1,23}=0 & \lambda_{2,13}=0, \\
\lambda_{1,24}-\frac{h_{23,2}}{h_{23}} \lambda_{1,4}=0 & \lambda_{2,14}-\frac{h_{13,1}}{h_{13}} \lambda_{2,4}=0, \\
\lambda_{1,34}-\frac{h_{23,3}}{h_{23}} \lambda_{1,4}=0 & \lambda_{2,34}-\frac{h_{13,3}}{h_{13}} \lambda_{2,4}=0, \\
\lambda_{1,24}-\frac{h_{23,2}}{h_{23}} \lambda_{1,4}=0 & \lambda_{2,14}-\frac{h_{13,1}}{h_{13}} \lambda_{2,4}=0 .
\end{array}
$$

From this two systems, we obtain that $\lambda_{1}$ and $\lambda_{2}$ are given by (26) and $\lambda_{3}, \lambda_{4}$ are obtained by substituting $\lambda_{1}$ and $\lambda_{2}$ in (31).

Finally, we will show that $L_{1}$ and $L_{2}$ satisfy equations (27). For this, we proceed in a similar way to obtaining (16). That is, using (4) and (5), we get

$$
\begin{aligned}
& {\left[L_{1}\left(\lambda_{2}-\lambda_{1}\right)^{2}\right]_{, 2}=0, \quad\left[L_{1}\left(\lambda_{2}-\lambda_{1}\right)^{2}\right]_{, 1}=0, \quad\left[L_{1}\left(\lambda_{j}-\lambda_{1}\right)^{2}\right]_{, j}=0,3 \leq j \leq 4,} \\
& {\left[L_{2}\left(\lambda_{4}-\lambda_{1}\right)^{2}\right]_{, 1}=0 .}
\end{aligned}
$$

Using the two first equations (32), we obtain $L_{1}\left(\lambda_{2}-\lambda_{1}\right)^{2}=\bar{f}_{34}$, where $\bar{f}_{34}$ is a differentiable function of $\left(x_{3}, x_{4}\right)$. Substituting (30) and (31) in this last equation and using the third equation (32), we have $\bar{f}_{34, j}=0,3 \leq j \leq 4$. Therefore, we obtain

$$
L_{1}=\left[\frac{c}{\lambda_{2}-\lambda_{1}}\right]^{2}
$$

where $c \neq 1$ is an arbitrary constant.

Now, using the last equation (32), we obtain $L_{2}\left(\lambda_{4}-\lambda_{1}\right)^{2}=\bar{f}_{234}^{2}$, where $\bar{f}_{234}$ is a differentiable function of $\left(x_{2}, x_{3}, x_{4}\right)$. Differentiating the last equation with respect $x_{2}$ and $x_{3}$, using (4), (5), and (31), we get

$$
\frac{\bar{f}_{234,2}}{\bar{f}_{234}}=\frac{h_{23,2}}{h_{23}}, \quad \frac{\bar{f}_{234,3}}{\bar{f}_{234}}=\frac{h_{23,3}}{h_{23}},
$$

where $h_{23}=f_{2}+f_{3}$.

Therefore, $\bar{f}_{234}=h_{23} f_{4}$, where $f_{4}$ is an arbitrary differentiable function of $x_{4}$. Thus, 
we have

$$
L_{2}=\left[\frac{h_{23} f_{4}}{\lambda_{4}-\lambda_{1}}\right]^{2} .
$$

Making $d \widetilde{x}_{1}=c d x_{1}, d \widetilde{x}_{2}=c d x_{2}, d \widetilde{x}_{3}=c d x_{3}$ and $d \widetilde{x}_{4}=f_{4} d x_{4}$, we obtain (27).

The next theorem provides a local characterization for $M^{r} \subseteq R^{r+1}, 3 \leq r \leq 4$, a 2-Isothermic proper Dupin hypersurface.

Theorem 8. Let $M^{r} \subseteq R^{r+1}, 3 \leq r \leq 4$, be a 2-isothermic proper Dupin hypersurface that is parametrized by lines of curvature and has $r$ distinct principal curvatures $-\lambda_{i}$ given by (15) if $r=3$, and by (26) if $r=4$. Then $M$ can be parametrized by

$$
X=\left\{\begin{array}{l}
\frac{1}{\lambda_{2}-\lambda_{1}}\left[G_{1}-G_{2}+\left(f_{1}-f_{2}\right) G_{3}\right], \text { if } r=3 \\
\frac{1}{\lambda_{2}-\lambda_{1}}\left[H_{1}-H_{2}+(c-1) H_{3}+\left(c f_{1}-f_{2}+(c-1) f_{3}\right) H_{4}\right], \text { if } r=4,
\end{array}\right.
$$

where $G_{i}$ and $H_{j} 1 \leq i \leq 3,1 \leq j \leq 4$ are, respectively, vector valued functions of $R^{4}$ and $R^{5}$.

Proof: We will show case $r=4$. The case $r=3$ is similar.

From the equation (3) using (10), we have

$$
\left(X,{ }_{4}-\Gamma_{14}^{1} X\right){ }_{, i}-\Gamma_{4 i}^{4}\left(X,_{4}-\Gamma_{14}^{1} X\right)=0, \quad 1 \leq i \leq 3 .
$$

This equation, using (4), (10), (31) and (30), we obtain

$$
X,_{4}=\Gamma_{14}^{1} X+\frac{\left(f_{2}+f_{3}\right) H_{4}^{\prime}}{\lambda_{1}-\lambda_{4}} .
$$

Again from the equation (3) using (10), we have

$$
\left(X,{ }_{3}-\Gamma_{13}^{1} X\right){ }_{, i}-\Gamma_{3 i}^{3}\left(X,_{3}-\Gamma_{13}^{1} X\right)=0, \quad 1 \leq i \leq 2 .
$$


This equation, using (4), (10), (31), (30) and (34), we obtain

$$
X,_{3}=\Gamma_{13}^{1} X+\frac{f_{3}^{\prime} H_{4}+H_{3}^{\prime}}{\lambda_{1}-\lambda_{3}} .
$$

Using (3) and (10), we get

$$
\begin{aligned}
& \left(X,_{2}-\Gamma_{12}^{1} X\right)_{, 1}-\Gamma_{21}^{2}\left(X,_{2}-\Gamma_{12}^{1} X\right)=0 \\
& \left(X,{ }_{1}-\Gamma_{12}^{2} X\right)_{, 2}-\Gamma_{21}^{1}\left(X,,_{1}-\Gamma_{12}^{2} X\right)=0 .
\end{aligned}
$$

Using this last two equations and (4), (10), (31), (30), (34) and (35), we obtain

$$
\begin{aligned}
& X,_{2}=\Gamma_{12}^{1} X+\frac{f_{2}^{\prime} H_{4}+H_{2}^{\prime}}{\lambda_{1}-\lambda_{2}}, \\
& X,_{1}=\Gamma_{12}^{2} X+\frac{c f_{1}^{\prime} H_{4}+H_{1}^{\prime}}{\lambda_{2}-\lambda_{1}} .
\end{aligned}
$$

Now, integrating the system determined by (34), (35), (36) and (37), using (31) and (30), we obtain

$$
X=\frac{1}{\lambda_{2}-\lambda_{1}}\left[H_{1}-H_{2}+(c-1) H_{3}+\left(c f_{1}-f_{2}+(c-1) f_{3}\right) H_{4}\right] .
$$

Following we provide an example of an irreducible 2-isothermic Dupin hypersurface with nonconstant Lie curvature, with four distinct principal curvatures. The used arguments in the construction of this example are analogous to those used in the construction of Example 4.2 in [13].

Example 9. Consider the constant $c=\frac{1}{2}$ and the functions

$$
\begin{aligned}
& f_{3}=\frac{-1}{2}+\cos \left(\sqrt{2} x_{3}\right), \quad f_{2}=\frac{1}{4} \sinh \left(x_{2}\right)+\frac{5}{4} \cosh \left(x_{2}\right)+\frac{1}{2}, \\
& h_{2}=\sinh \left(x_{2}\right)-\cosh \left(x_{2}\right)-\frac{1}{2}, \quad, f_{1}=\frac{1}{2}, \quad h_{3}=-f_{3}, \\
& h_{1}=\frac{-1}{2}-\frac{1}{2} \cos \left(\sqrt{2} x_{1}\right), \quad h_{4}=1+\frac{11}{8} \sinh \left(x_{4}\right)+\frac{15}{8} \cosh \left(x_{4}\right) .
\end{aligned}
$$


Now, define the functions $\lambda_{i}: U \subseteq R^{4} \rightarrow R$, where $U$ is a neighborhood of $(0,0,0,0)$

$$
\begin{aligned}
\lambda_{1} & =\left(f_{2}+f_{3}\right) h_{4}+h_{3}+h_{2}, \\
\lambda_{2} & =c\left[\left(f_{1}+f_{3}\right) h_{4}+h_{3}+h_{1}\right], \\
\lambda_{3} & =\frac{c}{1-c}\left[\left(f_{1}-f_{2}\right) h_{4}+h_{1}-h_{2}\right], \\
\lambda_{4} & =\frac{c\left[\left(f_{2}-f_{1}\right) h_{3}-\left(h_{2}-h_{1}\right) f_{3}+f_{2} h_{1}-f_{1} h_{2}\right]}{f_{2}-c f_{1}+(1-c) f_{3}}, \\
L_{1}= & \frac{1}{\left(\lambda_{2}-\lambda_{1}\right)^{2}}, \quad L_{2}=\left[\frac{f_{2}-c f_{1}+(1-c) f_{3}}{\lambda_{2}-\lambda_{1}}\right]^{2} .
\end{aligned}
$$

Consider the quadratic forms

$$
I=L_{1}\left(d x_{1}^{2}+d x_{2}^{2}+d x_{3}^{2}\right)+L_{2} d x_{4}^{2}, \quad I I=L_{1}\left(\lambda_{1} d x_{1}^{2}+\lambda_{2} d x_{2}^{2}+\lambda_{3} d x_{3}^{2}\right)+L_{2} \lambda_{4} d x_{4}^{2} .
$$

After very long computations we can show that these functions satisfy Gauss Equation (9) and Coddazi Equation (4). Therefore, by the Fundamental Hypersurfaces Theorem, there is a hypersurface whose first and second quadratic forms are given by (40). As the functions $\lambda_{i}$ above, satisfy $\frac{\partial \lambda_{i}}{\partial x_{i}}=0$ for all $1 \leq i \leq 4$, we have that the hypersurface is a Dupin type and by (40) is 2-isothermic hypersurface with principal curvatures $-\lambda_{i}$.

Using the Theorem 8, this hypersurface can be parametrized by

$$
X=\frac{1}{\lambda_{2}-\lambda_{1}}\left[H_{1}-H_{2}-\frac{1}{2} H_{3}+\left(\frac{1}{2} f_{1}-f_{2}-\frac{1}{2} f_{3}\right) H_{4}\right] .
$$

Given initial conditions

$X, i(0,0,0,0)=\frac{121 e_{i}}{32} \quad 1 \leq i \leq 3, \quad X,_{4}(0,0,0,0)=\frac{121 e_{4}}{56}, \quad N(0,0,0,0)=e_{5}$, 
and using (2) and (7), we have that vector functions $H_{i}$ above are given by

$$
\begin{aligned}
& H_{1}=\left(\frac{\sqrt{2}}{2} \sin \left(\sqrt{2} x_{1}\right), \frac{-5}{22} \cos \left(\sqrt{2} x_{1}\right), 0, \frac{-2}{11} \cos \left(\sqrt{2} x_{1}\right), \frac{-13}{22} \cos \left(\sqrt{2} x_{1}\right)\right) \\
& H_{2}=\left(0, \sinh \left(x_{2}\right)+\frac{5}{11} \cosh \left(x_{2}\right), 0, \frac{4}{11} \cosh \left(x_{2}\right), \frac{2}{11} \cosh \left(x_{2}\right)\right), \\
& H_{3}=\left(0, \frac{-5}{11} \cos \left(\sqrt{2} x_{3}\right), \sqrt{2} \sin \left(\sqrt{2} x_{3}\right), \frac{-4}{11} \cos \left(\sqrt{2} x_{3}\right), \frac{9}{11} \cos \left(\sqrt{2} x_{3}\right)\right) \\
& H_{4}=\left(1-\cosh \left(x_{4}\right)\left(0, \frac{-6}{11}, 0, \frac{-7}{11}, \frac{2}{11}\right)+\sinh \left(x_{4}\right)(0,0,0,1,0) .\right.
\end{aligned}
$$

Its Lie curvature (8) is given by

$$
\Psi=\frac{5 \cosh \left(x_{2}\right)+\sinh \left(x_{2}\right)}{\sinh \left(x_{2}\right)+5 \cosh \left(x_{2}\right)+4 \cos \left(\sqrt{2} x_{3}\right)} .
$$

Moreover, one can show that this is an irreducible Dupin hypersurface. In fact, we consider the stereographic projection of X into the unit sphere $S^{5} \subseteq R^{6}$, given by

$$
f=\gamma\left(X+\frac{|X|^{2}-1}{2} e_{6}\right), \quad \gamma=\frac{2}{|X|^{2}+1}
$$

where $X=X\left(x_{1}, x_{2}, x_{3}, x_{4}\right) \in U \subseteq R^{4}$ and $e_{6}=(0,0,0,0,0,1) \in R^{6}$. Let $\xi$ be the field of unitary normal vectors of the immersion $f$ given by $\xi=\langle X, N\rangle$ $\left(-f+e_{6}\right)+N$, where $\mathrm{N}$ is the unit normal vector field of the immersion $X \in R^{4}$. The pair $(f, \xi)$ induces a Legendre manifold $\mu: U \subseteq R^{4} \rightarrow \Lambda^{9}$, where $\Lambda^{9}$ is the manifold of lines that lie on the quadric $Q^{6}$, in the real projective space $P^{7}$, given by the equation $\langle<Y, Y\rangle>=0$, where $\langle<,>>$ is the scalar product with signature $(6,2)$ on $R^{8}$ (see [4] for details).

According to Pinkall [18], a proper Dupin submanifold $M^{n-1} \subseteq \Lambda^{2 n-1}$ is reducible if, and only if, it has a curvature sphere map $\mathrm{K}$ that lies in a linear subspace of codimension two in $P^{n+2}$. The curvature sphere maps of this example are given by

$$
K^{i}=\sin (t)\left[\widetilde{\lambda}_{i}(1, f, 0),(0, \xi, 1)\right], \quad 1 \leq i \leq 4
$$

where $\widetilde{\lambda}_{i}=\frac{\lambda_{i}}{\gamma}-\langle X, N\rangle$. The determinant of the $8 \times 8$ matrix, obtained by considering the first and second order derivatives $\frac{\partial K^{i}}{\partial x_{j}}$ and $\frac{\partial^{2} K^{i}}{\partial x_{j}^{2}}, 1 \leq j \leq 4$, evaluated 
at the point $(0,0,0,0)$ is nonzero. Hence, for any $i$, the curvature sphere map $K^{i}$ does not lie in a linear subspace of codimension two in $P^{7}$. Therefore, $X$ is an irreducible Dupin hypersurface.

Remark 10. The futures works will be in the directions of obtaining examples of 2-irredutible hypersurfaces that are not necessarily Dupin hypersurfaces.

\section{References}

[1] BIANCHI, L. Lezioni di Geometria Differenziale. Terza Edicione. Bologna: Nicola Zanichelli Editore, 1927.

[2] CAlapsO, P. Sulle superficie a linee di curvatura isoterme, Palermo Rend., v. 17, p. 257-286, 1903.

[3] CARTAN, E. La déformation des hypersurfaces dans l'espace conforme reel à $n \geq 5$ dimensions, Bull. Soc. Math. France, v. 45, p. 57-121, 1917.

[4] CECIL, T. E.; CHERN, S. S. Dupin submanifolds in Lie sphere geometry, Differential Geometry and Topology 1-48, Lecture Notes in Math. 1369, Springer, 1989.

[5] CIEŚLIŃSKI, J.; GOLDSTEIN, P.; SYM, A. Isothermic surfaces as solutions of Calapso PDE, Balkan J. Geom. Appl., v. 13, n. 1, p. 20-26, 2008.

[6] CIEŚLIŃSKI, J. The Darboux-Bianchi transformations for isothermic surfaces. Classical results the solitons approach, Differential Geom. Appl., v. 7, n. 1, p. 1-28, 1997.

[7] CORRO, A. M. V.; FERREIRA, W. P ; TENENBLAT, K. On Ribaucour transformations for hypersurfaces, Mat. Contemp., v. 17, p. 137-160, 1999.

[8] CORRO, A. M. V.; RIVEROS, C. M. C. Classes of hypersurfaces with vanishing Laplace invariants, Bull. Korean Math. Soc., v. 49, n. 4, p. 685-692, 2012.

[9] Corro, A. M. V.; FernandeS, K. V.; RIVEROS, C. M. C. Isothermic surfaces and solutions of the Calapso equation, Serdica Math. J., v. 44, p. 341-364, 2018. 
[10] DARBOUX, G. Sur les surfaces isothermiques, C. R. Acad. Sci. Paris, v. 128, p. 1299-1305, 1899.

[11] DARBOUX, G. Leçons sur la Théorie Générale des Surfaces et Applications Géométriques du Calcul Infinitesimal. New York: Chelsea Pub. Co, 1972.

[12] FERRO, M. L.; RODRIGUES, L. A.; TENENBLAT, K. On a class of Dupin hypersurfaces in $\mathbb{R}^{5}$ with nonconstant Lie curvature, Geometriae Dedicata, v. 67, p. 1-26, 2013.

[13] FERRO, M. L.; RODRIGUES, L. A.; TENENBLAT, K. On Dupin hypersurfaces in $\mathbb{R}^{5}$ parametrized by lines of curvature, Results Math., v. 70, p. 499-531, 2016.

[14] HERTRICH-JEROMIN U. Introduction to Möebius Differential Geometry. Cambridge: Cambridge University Press, 2003. v. 300. (London Mathematical Society Lecture Note Series)

[15] HERTRICH-JEROMIN U. On conformally flat hyupersurfaces and Guichard's nets, Beiträge Algebra Geom., v. 35, p. 315-331, 2003.

[16] HERTRICH-JEROMIN U.; SUYAMA Y. Conformally flat hypersurfaces with cyclic Guichard's net, Internat. J. Math., v. 18, p. 301-329, 2007.

[17] LAME G. Leçons sur les Coordonnés Curvilignes et leurs Diverses Applications. Paris: Mallet-Bachelier, 2007.

[18] PINKALL, U. Dupin hypersurfaces, Math. Ann., v. 270, p. 427-440, 1985.

[19] RIVEROS, C. M. C. Dupin hypersurfaces with four principal curvatures in $\mathbb{R}^{5}$ with principal coordinates, Rev. Mat. Complut., v. 23, n. 2, p. 341-354, 2010.

[20] RIVEROS, C. M. C. A characterization of Dupin hypersurfaces in $\mathbb{R}^{4}$, Bull. Belg. Math. Soc. Simon Stevin, v. 20, n. 1, p. 145-154, 2013.

[21] RIVEROS, C. M. C. A characterization of Dupin hypersurfaces in $\mathbb{R}^{5}$, Serdica Math. J., v. 43, n. 2, p. 169-186, 2017. 
[22] Riveros, C. M. C.; TENEnBlat, K. Dupin hypersurfaces in $\mathbb{R}^{5}$, Canad. J. Math., v. 57, n. 6, p. 1291-1313, 2005.

[23] Riveros, C. M. C.; ROdRIGUeS, L. A.; TenenBlat, K. On Dupin hypersurfaces with constant Möebius curvature, Pacific J. Math., v. 236, n. 1, p. 89-103, 2008.

[24] SANTOS, J.; TENENBLAT, K. The symmetry group of Lamé's system and the associated Guichard nets for conformally flat hypersurfaces, SIGMA Symmetry Integrability Geom. Methods Appl., v. 9, Paper 033, 27 pp, 2013.

[25] SUYAMA Y. Conformally flat hypersurfaces in Euclidean 4-space, Nagoya Math. J., v. 158, p. 1-42, 2000.

[26] SUYAMA Y. Conformally flat hypersurfaces in Euclidean 4-space II, Osaka J. Math., v. 42, p. 573-598, 2005.

[27] SUYAMA Y. Conformally flat hypersurfaces in Euclidean 4-space and a class of Riemannian 3-manifolds, Sūrikaisekikenkyūsho kōkȳuroku, v. 1236, p. 60-89, 2001.

[28] YOUnG, A. E. On certain isothermic surfaces, Trans. Amer. Math. Soc., v. 8 , n. 3 , p. $415-426,1907$.

Submetido em 03 Out. 2019

Aceito em 05 Dez. 2019 